\section{Cahiers de Narratologie}

Analyse et théorie narratives

$10.1 \mid 2001$

La voix narrative

\title{
« La voix de son maître »
}

La construction du lecteur par le discours auctorial dans Les Etudes de mœurs de Balzac

\section{Christèle Couleau}

\section{(2) OpenEdition \\ 1 Journals}

\section{Electronic version}

URL: http://journals.openedition.org/narratologie/6959

DOI: 10.4000/narratologie.6959

ISSN: 1765-307X

\section{Publisher}

LIRCES

\section{Printed version}

Date of publication: 1 January 2001

Number of pages: 327-334

ISBN: 2914561032

ISSN: 0993-8516

\section{Electronic reference}

Christèle Couleau, "«La voix de son maître »", Cahiers de Narratologie [Online], 10.1 | 2001, Online since 28 October 2014, connection on 23 February 2021. URL: http://journals.openedition.org/narratologie/ 6959 ; DOI: https://doi.org/10.4000/narratologie.6959 


\title{
« LA VOIX DE SON MAÎTRE » \\ La construction du lecteur par le discours auctorial dans Les Etudes de mours de Balzac
}

\author{
Christèle COULEAU \\ Université de Caen
}

Chez Balzac, la voix narrative s'autorise des échappées discursives, prenant la forme "didactique d'un commentaire autorisé de l'action » et postulant «à la fois la présence de l'auteur (réel ou fictif) et l'autorité souveraine de cette présence dans son œuvre »'. Le réalisme balzacien repose moins sur l'illusion mimétique que sur ce mode autoritaire de distribution du savoir et du sens : c'est le principe même du 《vraisemblable artificiel $»^{2}$. Dans ce système, nous dit Philippe Hamon, « le lecteur ne cro[it] à ce qui est dit que s'il croit en celui qui dit $»^{3}$. Le discours auctorial ne se contente donc pas de transmettre du sens, il tente aussi de conditionner cette transmission afin de la rendre optimale, il forme son destinataire tout autant qu'il l'informe. Dans l'idéal balzacien, lire, ce serait donc avant tout reconnaître dans le texte la voix de son maître.

\section{Stratégies et postures : la construction du lecteur}

Ce désir de maîtriser ce que par essence le texte ne maîtrise plus, conduit Balzac à construire la figure d'un lecteur ad hoc - lecteur inscrit, impliqué, fictionnalisé, distinct du lecteur réel qui reste libre de se reconnaître ou non dans ce miroir que lui tend le texte. Balzac interpelle ce lecteur dans ses romans et lui donne même parfois la parole. Pourtant

${ }^{1}$ Gérard GENETTE, Figures III, Seuil, p. 263-264.

2 Figures II, Points Seuil, p. 79.

3 Cf. Philippe HAMON, "L'architecture/Le sens/Le réel/ La représentation ", in Roman, réalités, réalisme. 
ce n'est guère l'indice d'une ouverture, d'un désir de dialogue : sa démarche correspondrait plutôt à la quête d'une complétude dévorante de l'œuvre qui en viendrait à englober celui-là même qui la lit. Ainsi que le souligne Franc Schuerewegen ${ }^{4}$ : "s'adresser au lecteur, c'est nécessairement se l'approprier, l'annexer à l'œuvre par le geste même qui l'interpelle ». Le narrataire apparaît dès lors comme une pure "stratégie textuelle $»^{5}$ au service des enjeux de la narration.

\section{L'adresse au lecteur : un conditionnement}

Il ne s'agit pas ici de dénombrer les procédés stylistiques de l'adresse au lecteur, déjà étudiés par Eric Bordas 6 , mais de voir comment, à travers ces adresses, Balzac essaie de se construire un lecteur adéquat. Umberto Eco a expliqué dans l'Apostille au Nom de la rose comment le texte peut, par sa forme même, se créer un lecteur correspondant à ses exigences. C'est de ce désir qu'il faut sans doute voir la trace dans l'utilisation systématique de l'impératif : " Imaginez », « Voyez », « Observez », "Comparez » dit sans cesse Balzac à son lecteur, comme pour mieux le faire entrer dans un système poétique dont le postulat majeur est sa curiosité pour le réel et sa représentation. Les effets de captatio benevolentiae, quant à eux, dessinent les contours de sa complaisance. Enfin, les reproches adressés à certains lecteurs pressés, blasés, ou trop focalisés sur les enjeux de la tension dramatique, sont un moyen de définir un lecteur idéal, capable de s'inscrire dans le rythme digressif choisi par le texte - bref, un lecteur endurant.

Ce conditionnement du lecteur se cristallise dans l'un des points stratégiques du système romanesque : la préface, dont Balzac a mis en fiction le fonctionnement dans Le Lys dans la vallée. Félix, écrivant à Natalie le récit de sa vie, accompagne cette missive d'une lettre d'envoi où il définit la réception qu'il attend : il s'agit donc d'une sorte de pacte de lecture, qui peut nous renseigner, métaphoriquement, sur le rapport qui unit Balzac à son lecteur. Or, qu'en ressort-il ? Imposant une interprétation canonique du texte, qu'il double

${ }^{4}$ Balzac contre Balzac, Sedes/Paratexte, p. 142.

5 Umberto Eco, Lector in Fabula, p. 76.

${ }^{6}$ Balzac, discours et détours. 
d'un appel à la compassion, Félix décline toute responsabilité en cas de lecture "fautive». Si la lectrice s'écarte de la «bonne » lecture, c'est à ses risques et périls : «S'il y avait dans cette confession des éclats qui te blessassent, souvienstoi que tu m'as menacé si je ne t'obéissais pas, ne me punis donc point de t'avoir obéi ? ». Menace contre menace, c'est bien une lecture contrainte qui s'esquisse ici, et qui pour l'instant bute encore sur l'écart existant entre lectrice réelle et lectrice idéale - écart que la suite du récit va tenter de résorber, multipliant les adresses à un lecteur qui s'éloigne de plus en plus de la personnalité de Natalie pour tendre vers un pur fantasme donnant lieu à de véritables mises en scènes narratives.

\section{Scénographies de la lecture}

Le discours auctorial travaille à se créer un simulacre de lecteur, qu'il met en scène afin de mieux se légitimer. Or, contrairement à ce qu'on pourrait attendre, la figure ainsi dessinée reste éminemment mouvante. Le lecteur balzacien n'est pas fixé, figé une fois pour toutes. Seulement, parmi toutes les identités et toutes les réactions possibles, Balzac aura soin de privilégier celles qui servent sa cause, et que l'on pourrait rassembler pour l'essentiel autour de trois figures emblématiques : l'élève, le contradicteur, l'alter ego : trois images, trois postures permettant une lisibilité maximale du texte.

- Le premier adjuvant de la poétique balzacienne est celui qui ne sait pas. Comment justifier la dimension didactique du roman si ce n'est en se supposant un lectorat novice qu'il va dès lors s'agir d'initier? Dans ces romans d'apprentissage du lecteur, le narrateur va décrire Paris au provincial, à l'aristocrate, les mœurs du commerce, aux honnêtes gens, les lois du bagne. Les a priori de ces lecteurs, leurs erreurs supposées, les questions rhétoriques que leur prête le narrateur seront autant d'embrayeurs du discours auctorial. Balzac forge ainsi un cercle vertueux qui participe pleinement à la dynamique narrative : le discours s'invente un lecteur qui réclame du discours, etc.

- Plus stimulant encore est le rapport qui oppose le narrateur à son hypothétique contradicteur, légitimant l'inflation expli- 
cative : il faut bien répondre, se justifier, expliquer ce qui n'a pas été compris. A travers ce lectorat rétif, c'est un défi que se lance le narrateur, mettant son discours à l'épreuve de l'autre-défi souvent faussé, le contradicteur mettant rarement en péril l'échafaudage textuel que sa présence doit renforcer ! Il en est ainsi lorsque le narrateur de La Vieille fille s'adresse soudain à certaines lectrices, des «femmes légères [qui] essaieront peut-être de chicaner la vraisemblance de ce récit ${ }^{7}$. Si le narrateur leur donne un instant la parole, c'est pour mieux opposer à leur logorrhée inefficace toute son autorité de spécialiste de la société française et de moralisateur catholique. L'attaque est minée de l'intérieur, son agressivité n'a d'égal que son caractère bénin. Mais cette diversion aura du moins permis de surprendre le lecteur - le vrai -, et de renforcer le narrateur dans ses prérogatives. Bref, ces mauvaises lectrices sont d'utiles lectrices, et le discours auctorial ressort nécessairement grandi de cette mise à l'épreuve fictive.

- Mais le narrateur peut aussi, sans faire le détour par la contestation, chercher à s'affirmer en accord avec le lecteur. Le rapport de forces cède alors la place à la complicité. La figure ainsi dessinée est celle d'un alter ego digne du discours qu'on lui destine. Apte à le comprendre, et même à le prolonger, il est pris à témoin de la vérité du texte : "Ah ! sachez-le : ce drame n'est ni une fiction, ni un roman. All is true, il est si véritable, que chacun peut en reconnaître les éléments chez soi, dans son cœur peut-être ${ }^{8}$. Placée au début du Père Goriot, cette apostrophe joue le rôle d'un pacte de lecture. Les frontières entre le récit et le réel sont gommées et le lecteur est convié à garantir la crédibilité du texte : son expérience de la vie, sa connaissance de lui-même sont l'aune à laquelle doit se mesurer l'authenticité du récit. Dans sa lecture doit se retrouver l'idée même qui a présidé à l'écriture : le souci de témoigner. $\mathrm{Ni}$ vrai (malgré les dénégations, cela reste une fiction), ni vraisemblable (ce n'est pas la mimesis qui est à l'œuvre ici), le texte est " véritable », c'està-dire vérifiable par l'expérience, propice à l'identification.

7 La Vieille fille, Garnier-Flammarion, p. 111.

${ }^{8}$ Le Père Goriot, Pléiade, II, p. 50. 
Ce type de lecteur, ici très peu spécifié compte-tenu de la valeur très globale de la remarque, peut être doté de compétences plus spécialisées selon les besoins du texte. Ainsi Balzac se construit un lecteur à son image, "[s]on semblable, [s]on frère ", toujours prêt à souscrire de bonne foi au discours auctorial.

\section{Diviser pour mieux régner?}

Apostropher le lecteur, c'est souvent l'inclure de force dans le texte, c'est aussi parfois l'exclure. Alors que certains lecteurs fictifs sont érigés en modèle, d'autres sont mis au pilori, exilés, interdits de lecture. Pourquoi ?

- Distribuer ainsi les bons points et les blâmes permet de hiérarchiser les lectures, et donc de valoriser une interprétation parmi d'autres, ce qui permet au narrateur de contrôler la réception et d'assurer l'univocité du texte. Souvent, d'ailleurs, les fautifs sont clairement désignés (les « femmes légères ») : ils servent ainsi de boucs-émissaires pour que la lecture, à côté, puisse suivre son cours. Cependant, souvent aussi, les mauvais lecteurs sont parmi nous, car leur désignation est assez vague pour inclure la majeure partie du lectorat, voire sa totalité. Dans La Femme de trente ans le narrateur explique ainsi qu'il "faudrait avoir l'âme de Julie pour comprendre... ». Or quel lecteur peut se vanter d'avoir l'âme de Julie ? Est-ce à dire qu'une partie du texte, irrémédiablement, nous échappe ?

- Ce second type d'exclusion, plus radical, permet au narrateur de valoriser ses propres capacités, et l'on peut gager qu'à travers ces lecteurs incompétents, c'est moins leur portrait qu'il dessine que le sien, en négatif. Si le lecteur est celui qui par définition «n'est pas » (de fait, il n'est pas Julie, ni un autre personnage de la fiction, ni l'architecte, le poète ou l'artiste qu'on réclame ici ou là), le narrateur est au contraire celui qui « est » : omniscient, il a effectivement l'âme de Julie (pensons à Flaubert : "Mme Bovary, c'est moi »). Mais cette valorisation risque de s'effectuer au prix d'une stérilisation du roman, refermé sur la seule expérience du narrateur.

- Ce serait compter sans les pouvoirs de l'identification. Les fréquents renvois d'une œuvre à l'autre doivent permettre au 
lecteur de combler les lacunes de son expérience par l'acquisition d'une expérience de substitution, acquise au sein de la fiction. A ce titre le roman est un élargissement de l'âme, semblable à ce vertige décrit par le narrateur de Facino Cane qui, dit-il « me donnait la faculté de vivre la vie de l'individu sur lequel elle s'exerçait [...] je pouvais épouser leur vie ; leurs désirs, leurs besoins, tout passait dans mon âme ». Vous n'avez pas encore l'âme de Julie, qu'à cela ne tienne : la lecture et la relecture de l'œuvre finiront par vous la donner, vous vivrez vous aussi de la vie d'autrui. On atteint là une sorte d'utopie romanesque, présente dans l'œuvre de Balzac, mais qui ne saurait cependant résumer sa poétique.

\section{Du lecteur déconstruit à la déconstruction du sens}

Passer de la construction fictive du lecteur à son incarnation réelle, c'est interroger l'efficacité du système narratif. Or à cette épreuve, le texte balzacien dévoile quelques ambiguités.

\section{La lecture comme prise de liberté}

La labilité de l'identité du lecteur fictif cache on l'a vu une intentionnalité narrative, et d'ailleurs, le livre est destiné à un lectorat hétérogène, irréductible à un seul narrataire. Cependant, le lecteur réel est en droit de s'interroger face à ce tourbillon protéifforme que le narrateur propose à son identification. Est-il vraiment capable de réaliser la synthèse idéale de toutes ces postures? La réponse est sans doute non. Le processus d'identification est alors mis en doute, et ce flottement ne peut qu'aller à l'encontre du souci d'univocité évoqué plus haut.

D'autre part, le motif de l'exclusion du lecteur, malgré l'esquisse d'une solution utopiste, pose problème au lecteur réel. On atteint un cas limite dans Mme Firmiani, où le narrateur, après avoir énuméré les conditions d'une lecture idéale, congédie celui qui n'y correspondrait pas : « autrement, [dit-il], vous jetteriez ce livre ici ». Le défi est ici d'autant plus dangereux que le texte se targue d'un pouvoir qu'il ne saurait exercer : se débarrasser d'un lecteur importun. Face à ce geste paradoxal du narrateur, le lecteur peut décider de passer outre. Franc Schuerewegen souligne ce paradoxe : 
« la nouvelle suscite, en raison, justement, de sa volonté d'exclure, l'avènement de lectures autres, d'autant plus possibles que le texte les prétend indésirables ${ }^{9}$. Le cas de Natalie, déjà évoqué, est à cet égard parlant. Refusant le fantasme du narrateur, elle se réapproprie in extremis son identité et revendique, dans sa lettre réponse, sa propre interprétation, bien éloignée des présupposés émis par Félix : Natalie, ou la revanche du lecteur réel sur le lecteur fictif !

Enfin, l'exclusion peut déboucher sur l'émulation. Dire au lecteur que son statut l'empêche d'accéder au sens du texte, c'est affirmer que le sens n'est pas entièrement donné à lire. Subsiste donc un certain mystère résiduel, qui peut s'interpréter comme un espace herméneutique offert à l'appétit du lecteur réel.

Paradoxalement, l'excès de maîtrise affirmé par le narrateur aboutit donc à la libération du lecteur. La tentation sera dès lors, pour reprendre l'expression de Franc Schuerewegen, de lire «Balzac contre Balzac». Lecture que Balzac repousse bien sûr globalement avec ostentation, mais qu'il dessine peut-être dans les détails de la narration?

\section{L'attente d'un lecteur actif}

Alors même qu'il met en place les conditions d'une lecture contrainte, le texte semble en attente d'une lecture autre, fondée à la fois sur l'observation et sur une ironie qui s'exerce notamment à l'encontre de l'autorité narratoriale. A la fin de La Vieille fille, c'est un narrateur quelque peu désabusé sur le rôle du roman qui énonce la morale de l'histoire : «Quand cette histoire n'aurait eu d'autre effet que d'inspirer aux possesseurs de quelques reliques adorées une sainte peur [...] elle aurait rendu d'énormes services». L'allusion réfère ici à la " triste » destinée de la tabatière du chevalier de Valois, symbole d'amour et de fidélité, qui se retrouve exposée dans un cabinet dévolu aux amours de passage. Cette notation tient davantage de la dérision que du propos sérieux : dérision vis-à-vis du personnage, moins pur qu'il ne le donnait à penser, mais aussi autodérision touchant le discours et ses objectifs didactiques. Le narrateur perd ici

${ }^{9}$ Balzac contre Balzac, op. cit., p. 19. 
de son autorité, mais c'est au bénéfice d'un creusement des significations. L'ironie permet ainsi de donner au lecteur un rôle dans l'élaboration du sens : comme l'explique Vladimir Jankelevitch, elle « sollicite l'intellection », elle est « un appel qu'il faut entendre ${ }^{10}$.

L'autre appel que doit entendre le lecteur sous les affirmations contradictoires du texte, est celui de l'observation. Les occurrences du verbe «lire » dans l'œuvre de Balzac concernent moins souvent la lecture d'un roman que celle des corps, des cœurs et des âmes. Si identification il y a, c'est peut-être là qu'elle se joue : le bon lecteur se doit de scruter le texte comme Balzac scrute le réel. A la limite, le vrai modèle du lecteur dans le roman ne serait pas le narrataire mais le narrateur lui-même.

L'obstination de Balzac à présenter son œuvre comme une «mosaïque » qu'il place sous l'égide de «Janus", montre qu'il est conscient de la polyphonie interne à son texte. Il lui faut dès lors un lecteur capable de déchiffrer le caché, de lire le multiple - non pour le synthétiser, mais pour en apprécier les justes anfractuosités, pour en emprunter les différents points de vue. Son texte est donc éminemment scriptible, et pour reprendre l'une des formules qu'il emploie dans La Physiologie du mariage, "lire, c'est créer peut-être à deux ». Quelle image du lecteur construit-il en définitive ? Celle d'un chien écoutant la voix de son maître ? Soit, mais pas n'importe lequel : encore faudrait-il prendre modèle sur le chien Couraut, qui, nous dit Balzac, " lisait la pensée de son maître dans ses yeux et la sentait exhalée dans l'aire de son corps ${ }^{11}$. Un chien-lecteur, chien-détective, apte à lire les indices du texte et à en réécrire le sens.

${ }^{10}$ L'Ironie, Champs-Flammarion, p. 64.

11 Une ténébreuse affaire, Pléiade. 